\title{
Effect of Modifying Signal Parameters on the Beamforming Performance in CDMA Mobile Communications
}

\author{
B. Banitalebi \\ Engineering Faculty of \\ Shiraz university
}

\author{
M. Karimi \\ Engineering Faculty of \\ Shiraz university
}

\author{
M. Kamarei \\ Engineering Faculty of \\ Tehran university
}

\author{
G. Dadashzadeh \\ Iran Telecommunication \\ Research Center
}

\begin{abstract}
In mobile communications, beamforming is one of the most efficient techniques to suppress undesired effects of the transmission channel. In a CDMA mobile communications system, there are some parameters such as PN code length of the CDMA signals and modulation type and order that usually are changed to increase the capacity and data bit rate. Variation of these parameters can change the beamformer performance.

In this paper, effect of changing these parameters on the beamformer performance is investigated both theoretically and by using simulations. These investigations show that the above parameters have different effects on the beamformer performance.
\end{abstract}

Index Terms - Transmission Channel, Adaptive Beamforming, Mobile Communications, GWSSUS, LS-CMA, CDMA.

\section{INTRODUCTION}

In mobile communications, transmission channel has undesired effects on the transmitted signals. These effects cause significant degradations in the performance of the beamforming algorithms by changing some signal parameters. It causes receiving the signal of each user in some paths with different time delays, phase and amplitude error, angular spread and etc. Since these errors are occurred because of non ideal mobile communications environment, their effects can not be controlled efficiently. In [1] the effects of these parameters on the beamformer performance are investigated in detail. But there is another kind of signal parameters which can efficiently change the beamformer performance and also can be controlled by transmitter or receiver. In this paper the effects of these parameters on the beamformer performance are investigated.

In the former works, usually the effects of the first kind of signal parameters such as multipath effect, amplitude and phase error, angular spread, and time delay are investigated. In [2] a mathematical analysis about the role of some transmission channel effects on the beamformer performance is presented. Jaska et al. analyzes the effect of transmission channel on the beam power pattern, especially on the nulls depth and position in [3].

There are a large variety of the beamforming algorithms which can be used to improve the quality of the data received by the Base Station (BS). Constant Modulus Algorithm (CMA) [4], [5], Decision Directed Algorithm (DDA) [6], Self Coherence Restoral Algorithm (SCORE) [7] and Code Gated Algorithm (CGA) [8] are the most popular beamforming algorithm in mobile communications. Also, there are some techniques which are suggested to improve the performance of these algorithms. Linear Constraint-Constant Modulus Algorithm (LC-CMA) [9], [10] is a modified version of CMA which increases the resistance of the beamformer against undesired channel effects by taking some constraints for the beamformer into account. Other references [11], [12] try also to improve the resistance of the beamformer against errors and distortions caused by transmission channel or increase the convergence speed of the beamformer.

On the other hand, one of the most important challenges in new mobile communications generations is providing higher data bit rate for larger number of users. In order to response this requirement, using longer Pseudo Noise (PN) codes in CDMA mobile communications, higher order modulations, and higher performance modulation techniques are suggested. Besides changing the capacity of mobile communications networks and rate of data bit rate, variation of these parameters change the beamformer performance, hard.

Despite the importance of the effects of the above parameters on the beamformer performance, especially for new mobile communications generations, there is no considerable work on this topic. This paper analyzes effects of variation of some signal properties such as PN code length and modulation type and order on the performance of the beamformer for two simple and mostly used modulation techniques. The effect of changing input Signal to Interference and Noise Ratio (SINR) on the beamformer performance is also investigated in this paper. These investigations are done both theoretically and by using simulations.

Because of some limitations of mobile handsets such as small dimensions, small battery, and etc and also because of the small power of the received signal by the BS, beamforming in the BS is more necessary and also more applicable than in the handsets. So in this paper it is assumed that beamforming is done in the BS and in uplink mode. The Least SquaresConstant Modulus Algorithm (LS-CMA) [13]- [14], which is 


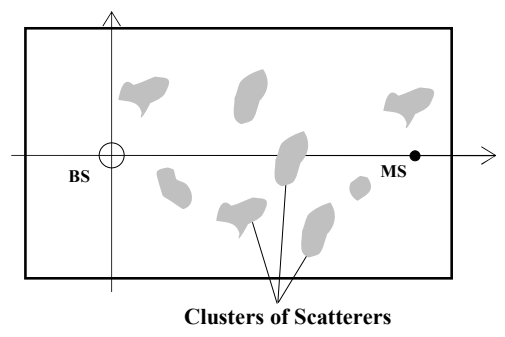

Fig. 1. A simple schematic of GWSSUS model

one of the CMA variants used least squares techniques to update beamforming weights, is used as the beamforming algorithm.

In Section II, signal and channel models are established. In section III after a discussion about the LS-CMA beamforming algorithm, a theoretical analysis is presented to show the effect of changing some controllable signal parameters on the beamformer performance. Results of the simulations are presented in section IV.

\section{SignAl AND CHANNEL MODELS}

In this paper, Gaussian Wide Sense Stationary Uncorrelated Scattering (GWSSUS) [15] is used as the transmission channel model. GWSSUS is a statistical channel model. In statistical channel models, some assumptions about the position and other properties of the scatterers are taken into account and all parameters of the signal are calculated based on these assumptions. In GWSSUS it is assumed that all scatterers are classified into some clusters, which are distributed uniformly between and around Mobile Sets (MSs) and BS. Also, the distribution of the scatterers in each cluster is uniform. These assumptions cause some special statistical distributions for different parameters of the signal received by the BS, which are taken into account in the signal model.

Fig. 1 shows a simple view of GWSSUS channel model. As seen in this figure, clusters of the scatterers are distributed randomly in the environment. In each environment, density and type of the scatterers and dimensions of the clusters depend on different environments. Therefore, the statistical distributions of signal parameters which represent transmission channel effects vary for different environments.

The complex envelope of data sequence of $i$-th mobile user at the channel input is as follows

$$
d_{i}(n)=a_{i}^{d}(n) e^{j \varphi_{i}^{d}(n)}
$$

where $a_{i}^{d}(n)$ and $\varphi_{i}^{d}(n)$ are the amplitude and phase of the $i$-th user in the $n$-th iteration, respectively. Note that $d_{i}(n)$ is the complex envelope of final form of signal after spreading, scrambling, and adding necessary codes such as channel codes.

The complex envelope of the received signal by the BS array antenna is

$$
\mathbf{x}=\sum_{i=1}^{U} \sum_{p=1}^{P} r_{i p}(n) \mathbf{a}\left(\theta_{i}+\delta_{i p}\right)+\mathbf{n}
$$

where $U$ is the number of users, $P$ is the maximum time delay which can be recovered by the receiver. $\theta_{i}$ is the angle of arrival of LOS ray of the $i$-th user and $\delta_{i p}$ is the angular spreading of $p$-th path of $i$-th user. $r_{i p}(n)$ is the signal of $p$-th path of the $i$-th user and $\mathbf{a}(\theta)$ is the steering vector correspond to direction $\theta$. Also, $\mathbf{n}$ is the $M \times 1$ additive white Gaussian noise vector. $r_{i p}(n)$ and $\mathbf{a}(\theta)$ can be written as

$$
\begin{aligned}
& r_{i p}(n)=\alpha_{i p}^{e} e^{j \varphi_{i p}^{e}} d_{i}\left(n-k_{i p}\right) e^{j \varphi_{i}^{o}} \\
& \mathbf{a}(\theta)=\left[\begin{array}{llll}
1 & e^{j 2 \pi \frac{d}{\lambda} \operatorname{Sin}(\theta)} & \cdots & e^{j 2 \pi(M-1) \frac{d}{\lambda} \operatorname{Sin}(\theta)}
\end{array}\right]^{T}
\end{aligned}
$$

where $k_{i p}$ is the time delay, $d$ is interelement spacing and $\lambda$ is the wavelength correspond to the central frequency of the transmitted signal. $\alpha_{i p}^{e}$ and $\varphi_{i p}^{e}$ are amplitude and phase error, respectively.

There are some paths of desired user whose time delays are larger than $P$. These paths are uncorrelated with the reference path (whose time delay is taken equal to zero) and act like interference signals. Since these signals usually pass a longer path, their power is too small and ignoring them does not have considerable effect on the signal model.

Based on GWSSUS model, $\alpha_{i p}^{e}$ 's of different paths of each user have Rician distribution when LOS rays exist, and Rayleigh distribution in non LOS cases. Also, $\varphi_{i p}^{e}$ 's are uniformly distributed in the interval [0 $2 \pi$ ). [15]

\section{TEORITICAL ANALYSIS}

The LS-CMA algorithm [13, 14] is one of the most popular beamforming algorithms in mobile communications. This algorithm generates beamforming weights as follows

$$
\mathbf{w}=\mathbf{R}_{x x}^{-1} \mathbf{r}_{x d}
$$

where $\mathbf{R}_{x x}$ and $\mathbf{r}_{x d}$, the autocorrelation matrix and cross correlation vector are

$$
\mathbf{R}_{x x}=E\left[\mathbf{x}(n) \mathbf{x}^{H}(n)\right]
$$

and

$$
\mathbf{r}_{x d}=E\left\lfloor\mathbf{x}(n) \hat{d}^{*}(n)\right\rfloor
$$

where $\mathbf{x}(n)$ and $\hat{d}(n)$ are the input vector and the training signal, respectively. This signal is correlated with the signal of desired user. In order to converge the beamformer to the proper weights, a known signal transmit at the beginning of the 


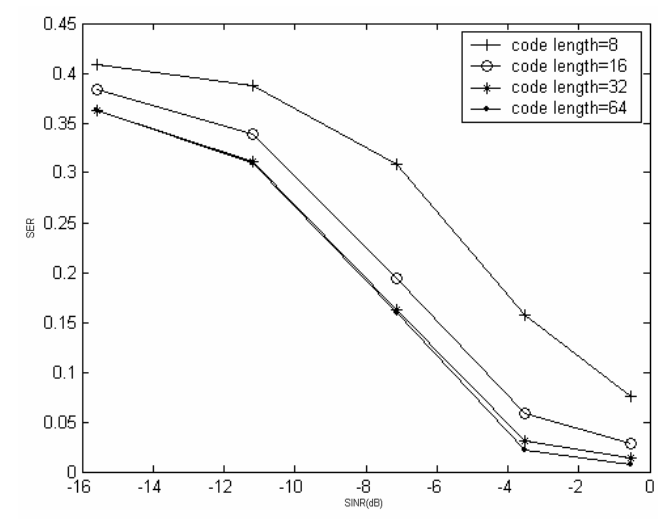

Fig. 2. Effect of changing PN code length

signal transmission. After convergence, the training signal is constructed by using the beamformer output. By using the signal model established in 2 , the autocorrelation matrix and cross correlation vector are calculated as follows

$$
\mathbf{R}_{x x}=\sigma_{s}^{2} \sum_{\mathrm{i}=1}^{U} \sum_{p=1}^{P} \rho_{i}\left(\theta_{i p}\right) \sigma_{e, i p}^{2} \mathbf{R}\left(\theta_{i p}\right)+\mathbf{R}_{n}
$$

and

$$
\mathbf{r}_{x d}=\sigma_{s}^{2} \sum_{p \in S} \rho_{1}\left(\theta_{1 p}\right) \mathbf{a}\left(\theta_{i p}\right)
$$

where $\rho_{1}(\theta)$ is the Probability Density Function (PDF) that describe the angular distribution of the desired signal paths, $\theta_{i p}$ is the angle of arrival of the $p$-th path of the $i$-th user, $\mathbf{R}(\theta)$ is the correlation matrix correspond with $\mathbf{a}(\theta)$, and $S$ is the set of paths of the desired signal which are correlated with the training signal because of their small time delays.

The above analysis show that the angular spread and multipath effect has no significant effect on the autocorrelation matrix but the cross correlation vector is affected by the angular spread, hard. The impact of the other effects of the transmission channel such as amplitude and phase error and time delay on the LS-CMA based beamformer is investigated in [1].

\section{SIMULATIONS}

There are some parameters of the signal which can be modified in the transmitter to reach better data rate or capacity. These parameters affect the beamformer performance. In this section effect of changing PN code length, and modulation type and order on the beamformer performance is investigated.

In the simulations of this section it is assumed that there are 7 homogeneous antenna elements with $\lambda / 2$ interelement spacing in the BS linear antenna array. BS antenna receives multipath signals from one desired user and 2 interfering mobile users. Desired and interfering signals are received in angular intervals around $0^{\circ}$ and $\pm 30^{\circ}$, respectively. The BS receives signal of each source from 15 paths. For each user, angles of arrival of these paths have truncated Gaussian distribution in a $40^{\circ}$ interval around the LOS direction. The delay for each path is a uniform random variable between 0 and 0.125 of bit length. It is assumed that the signal of each user is spreaded using a Walsh code. Both PSK and QAM modulation techniques are considered in the following simulations.

There are some parameters which can be used to represent beamformer performance such as output signal to noise and interference ratio $\left(\mathrm{SINR}_{\text {out }}\right)$, bit error rate (BER), and sample error rate (SER). Since in the simulations of this paper digital signals are used, BER or SER are better parameters for representation of beamformer performance. BER is calculated after despreading and represents improvements caused by both despreading and beamforming, while SER is calculated before despreading and shows improvement caused by beamforming only. So we use SER to represent beamformer performance. Our simulations are derived by using 1000 independent simulation runs of 200 data bits. In each simulation run all random signal parameters (amplitude, phase, and time and angle of arrival of each path) are changed according to their corresponding distributions.

Fig. 2 shows the effect of increasing PN code length on the output SER. As seen in this figure, increasing the code length improve the beamformer performance by decreasing the output SER. It is mainly because of increasing the peak autocorrelation to cross correlation ratio for the Walsh code when the code length increases. So, using longer spreading code causes smaller output SER. This point is also shown in Fig. 3. In this figure, the effect of increasing the code length on the symbol constellation for 8PSK signals is illustrated. As seen in this figure, when the code length increases, the ability of the beamformer to collect the received signal around their correct positions in the symbol constellation will increase.

Another point that can be achieved from Fig. 2 is about the difference between the curves. The difference between curves of small code length (e.g. 8 and 16) is more than the difference between curves of long codes (e.g. 32 and 64). Also it can be seen that increasing the SINR decrease the difference between the curves correspond to different code length. This decrease shows that the performance of the spreading codes improves in the case of higher SINRs.

Of course, the code length is usually increased to raise the number of users. So, because of the random position of the users in the cell, accurate analysis of the effect of increasing the code length on the output SER by simulation is so difficult. But in the case of fixed number of users, Fig. 2 shows the effect of increasing the code length. The most important limitation to increase the code length is the frequency bandwidth which transmitter can use.

Table I shows effects of changing signal parameters on the SER of the beamformer output. The first row of this table represents the effect of increasing the modulation order on the output SER. Increasing the modulation order arise the 


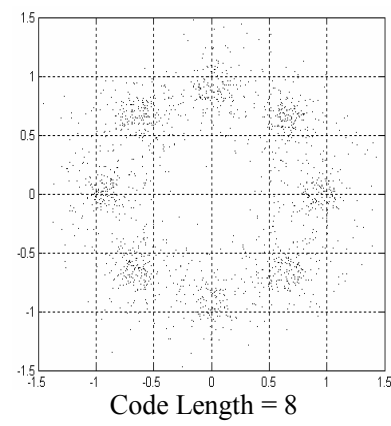

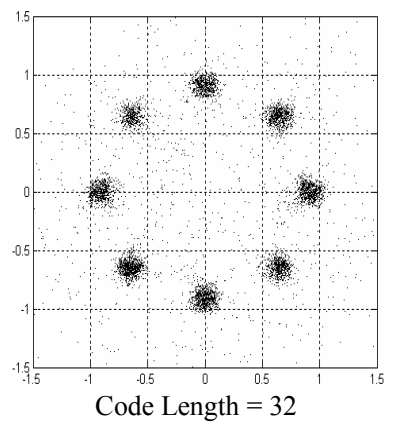

Code Length $=32$

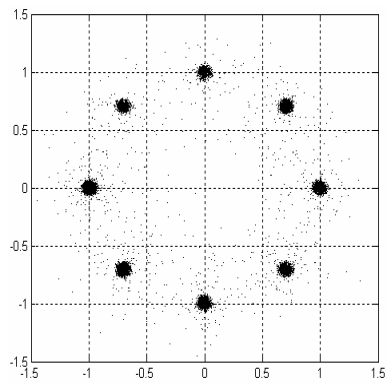

Code Length $=64$

Fig. 3. Symbol constellation of the output of the beamformer for 8PSK modulation with different code lengths. Increasing the code length increase the ability of the beamformer to collect symbols around their correct positions.

TABLE I

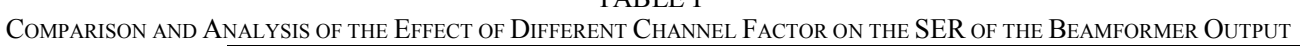

\begin{tabular}{|c|c|c|c|c|c|c|c|c|c|}
\hline & & & & & & & & & \\
\hline \multirow{2}{*}{ Order of Modulation } & Mod. Order & 16 & 8 & 4 & 2 & 32 & 16 & 8 & 4 \\
\hline & SER & 0.0710 & 0.0557 & 0.0370 & 0.0231 & 0.0809 & 0.0675 & 0.0585 & 0.0370 \\
\hline \multirow{2}{*}{$\begin{array}{c}\text { Signal to Interference } \\
\text { Ratio }^{\mathbf{a}}\end{array}$} & $\mathrm{S} / \mathrm{I}$ ratio & -10 & 0 & 10 & 20 & -10 & 0 & 10 & 20 \\
\hline & SER & 0.0169 & 0.0166 & 0.0166 & 0.0170 & 0.0119 & 0.0121 & 0.0121 & 0.0116 \\
\hline
\end{tabular}

a: the ratio of desired user power to each interferer power

capacity, considerably but also cause some degradation in the beamformer performance. The main cause of this degradation is the increased sensitivity of the signal against transmission channel effects. As seen in Fig. 4, increasing the order of modulation causes different symbols to approach each other in the symbol constellation. It also increases the SER of the received data by the receiver. In this case, the correlation between the received signal and training sequence decreases hard.

Another analysis that is presented in table I is a comparison between PSK and QAM modulation techniques.

In QAM, symbols are separated both in phase and in amplitude but in PSK, symbols are separated only in phase. Although the more complexity of the QAM detectors than the PSK cetectors, QAM is more resistant again transmission

channel effects. Simulation results in table I shows that the output SER in the case of QAM signals are smaller than the case of using PSK signals for unique order.

The second row of table I shows the effect of signal to interference $(\mathrm{S} / \mathrm{I})$ ratio on SER. It can be seen from this row that the $\mathrm{S} / \mathrm{I}$ ratio has no significant effect on the output SER both for QAM and PSK modulation techniques, at least for the range of S/I values investigated in this row. Similar to the first row, values of the output SER calculated for QAM signals are smaller than what calculated for PSK signals.

It is because QAM modulation symbols are separate both in phase and amplitude; but in PSK modulation symbols have the same amplitude and are separate only in phase. Achieving better quality by using QAM modulation is at the expense of more complex modulator and detector in transmitter and receiver, respectively.

\section{CONCLUSION}

Simulation results show that when the number of users is constant, increasing the code length can efficiently improve the performance of the beamformer. Increasing the modulation order to achieve higher data transmission rates causes some degradation in the output SER but it is not very serious. It was also shown that the signal to interference ratio has no significant effect on the beamformer performance. Another result which can be derived from the simulations is that using QAM modulation which uses both phase and amplitude to separate different symbols from each other gives smaller values of SER than PSK modulation.

\section{ACKNOWLEDGMENT}

Authors of this paper would like to thanks Iran Telecommunication Research Center for its supports and helpful contributions.

\section{REFERENCES}

[1] B. Banitalebi, M. Kamarei, M. Karimi, and G. R. Dadashzadeh, "Effects of the Transmission Channel on the LS-CMA Based Beamformer in Mobile Communications," to be accepted in Antem 2005.

[2] A. J. van der Veen, "Algebraic Methods for Deterministic Blind Beamforming," Proceeding of the IEEE, vol. 86, no. 10, Oct. 1998.

[3] E. A. Jaska, L. E. Corey and S. Y. Park, "Effects of Random Amplitude and Phase Errors on Monopulse Null Depth in Phased-Array Antennas," Proceeding of Antenna and Propagation Society International Symposium, 1990, AP-S. Merging Technology for the 90's Digest.

[4] D. N. Godard, "Self-recovering equalization and carrier tracking in twodimensional data communication system," IEEE Trans. Commun., vol. COMM-28, Nov. 1980. 

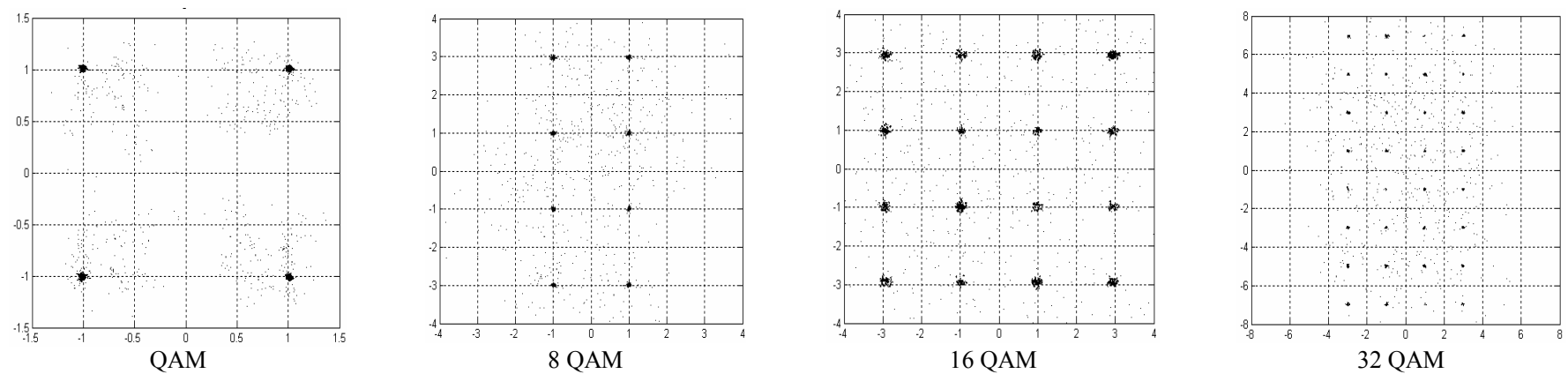

16 QAM

32 QAM

Fig. 4. Symbol constellation of the output of the beamformer for different orders of QAM modulation

[5] J. R. Triechler and B. G. Agee, "A new approach to multipath correction of constant modulus signals," IEEE Trans. Acoust., Speech, Signal Processing, vol. ASSP-31, Apr. 1983.

[6] A. L. Swindlehurst, S. Daas, and J. Yang, "Analysis of Decision Directed Beamformer," IEEE Trans. Signal Processing, vol. 43, no. 12, Dec. 1995.

[7] B. G. Agee, S. V. Schell, W. A. Gardner, "The SCORE Aproach to Blind Adaptive Signal Extraction: An Application of the Theory of Spectral Correlation,” IEEE Trans. Signal Processing, vol. 49, no. 5, Mar. 1999.

[8] 'Y. M. Vasavada, T. E. Biedka, and J. H. Reed, "Code Gated Algorithm: A Blind Adaptive Antenna Array Beamforming Scheme for the Wideband CDMA System," IEEE Trans. Signal Processing, vol. 45, no. 5, Jul. 1999.

[9] J. Miguez and L. Castedo, "A Linearly Constrained Constant Modulus Approach to Blind Adaptive Multiuser Interference Suppression," IEEE Communications Letters, vol. 2, no. 8, Aug. 1998.

[10] Z. Xu and P. Liu, "Constraint CMA-Based Multiuser Detection under Unknown Multipath," $12^{\text {th }}$ IEEE international Symposium on Personal, Indoor and Mobile Radio Communication, vol. 1, Oct. 2001.
[11] H. Liu and G. Xu, "Closed-Form Blind Symbol Estimation in Digital Communications," IEEE Trans. Signal Processing, vol. 43, Nov. 1995.

[12] A. J. van der Veen, S. Talwar, and A. Paulraj, "A Subsequence Approach to Blind Space-Time Signal Processing for Wireless Communication systems," IEEE Trans. Signal Processing, vol. 45, Jan. 1997

[13] B. Agee, "The Least Squares CMA: A New Technique for Rapid Correction of Constant Modulus Signals," Acoustics, Speech, and Signal Processing, IEEE International Conferences on ICASSP '86, vol. 11, Apr. 1986.

[14] T. E. Biedka, W. H. Tranter, and J. H. Reed, "Convergence Analysis of the Least Squares Constant Modulus Algorithm," 1996 Conference Record of the Thirtieth Asilomar Conference on Signal, Systems and Computers, vol.1, Nov. 1996.

[15] U. Martin and M. Gerigat, "A Statistical Simulation Model for Directional Mobile Radio Channel and its Configuration," International Symposiem on Spread Spectrum Techniques and applications proceedings, vol. 1, Sep. 1996. 
